Review Article

\title{
Strong Public Health Recommendations from Weak Evidence? Lessons Learned in Developing Guidance on the Public Health Management of Meningococcal Disease
}

\author{
Germaine Hanquet, ${ }^{1,2}$ Pawel Stefanoff, ${ }^{3,4}$ Wiebke Hellenbrand, ${ }^{5}$ Sigrid Heuberger, 6 \\ Pierluigi Lopalco, ${ }^{7}$ and James M. Stuart ${ }^{8,9}$ \\ ${ }^{1}$ Consultant Epidemiologist (Independent), 1081 Brussels, Belgium \\ ${ }^{2}$ Department of Vaccinology, University of Antwerp, 2000 Antwerp, Belgium \\ ${ }^{3}$ Division of Epidemiology, Norwegian Institute of Public Health, 0403 Oslo, Norway \\ ${ }^{4}$ National Institute of Public Health-National Institute of Hygiene, 400-791 Warsaw, Poland \\ ${ }^{5}$ Department of Infectious Disease Epidemiology, Immunization Unit, Robert Koch Institute, 13086 Berlin, Germany \\ ${ }^{6}$ Meningococcal Reference Laboratory, Austrian Agency for Food and Health Safety, 8010 Graz, Austria \\ ${ }^{7}$ Vaccine-Preventable Diseases Programme, European Centre for Disease Prevention and Control, 17183 Stockholm, Sweden \\ ${ }^{8}$ School of Social and Community Medicine, University of Bristol, Bristol BS8 2BN, UK \\ ${ }^{9}$ Faculty of Infectious and Tropical Diseases, London School of Hygiene \& Tropical Medicine, London WC1E 7HT, UK \\ Correspondence should be addressed to Pawel Stefanoff; pawel.stefanoff@gmail.com
}

Received 5 June 2015; Revised 22 September 2015; Accepted 26 October 2015

Academic Editor: Malgorzata Bala

Copyright (C) 2015 Germaine Hanquet et al. This is an open access article distributed under the Creative Commons Attribution License, which permits unrestricted use, distribution, and reproduction in any medium, provided the original work is properly cited.

The evidence underpinning public health policy is often of low quality, leading to inconsistencies in recommended interventions. One example is the divergence in national policies across Europe for managing contacts of invasive meningococcal disease. Aiming to develop consistent guidance at the European level, a group of experts reviewed the literature and formulated recommendations. The group defined eight priority research questions, searched the literature, and formulated recommendations using GRADE methodology. Five of the research questions are discussed in this paper. After taking into account quality of evidence, benefit, harm, value, preference, burden on patient of the intervention, and resource implications, we made four strong recommendations and five weak recommendations for intervention. Strong recommendations related not only to one question with very low quality of evidence as well as to two questions with moderate to high quality of evidence. The weak recommendations related to two questions with low and very low quality of evidence but also to one question with moderate quality of evidence. GRADE methodology ensures a transparent process and explicit recognition of additional factors that should be considered when making recommendations for policy. This approach can be usefully applied to many areas of public health policy where evidence quality is often low.

\section{Introduction}

The incidence of invasive meningococcal disease (IMD), caused by Neisseria meningitidis, is low in Europe, but case fatality is high ( 0.8 cases $/ 100,000$ inhabitants and $8.7 \%$, resp., in 2011) [1]. Outbreaks of IMD may generate significant anxiety in the population, and even a single case may have important public health implications $[2,3]$. N. meningitidis is transmitted from person to person and the risk of disease is highest in contacts from the same household as a case $[4,5]$. In 2007, a European survey showed that recommendations for chemoprophylaxis to eliminate nasopharyngeal carriage in close contacts of IMD cases varied across Europe, in particular regarding the type of antibiotic and the groups that should be targeted $[6,7]$. Discrepancies were partly due to differences in policy, medical practices, and health systems 
but could also be explained by uncertainty surrounding the effectiveness of preventive measures or the differences in methods used to develop recommendations [6].

Divergences in national policies are particularly problematic in cross-border settings, as they lead to differences in disease management among population groups with the same exposure. For instance, passengers sharing an aeroplane flight with a case of IMD might or might not receive chemoprophylaxis depending on their country of residence [8]. The European Centre for Disease Prevention and Control (ECDC) therefore commissioned a group of experts to develop guidance for European countries on the management of contacts of IMD.

It was clear from the outset that high quality evidence in this area would be limited as in many areas of public health [9]. This is because randomized clinical trials on public health interventions are often difficult to organize (particularly when incidence of the outcome is low) and the use of placebo is no longer considered ethical when the intervention studied is already a recommended standard of care. In addition, evidence may be indirect as when only surrogate (proxy) endpoints are available. Therefore, information often comes from observational studies that are more prone to bias and are considered to provide a lower quality of evidence [10]. However, such studies can still be used in developing recommendations if systematically researched and graded appropriately [11]. In addition, evidence obtained for a public health intervention in one country may not be fully applicable to another setting, as public health interventions are strongly dependent not only on the epidemiological context but also on cultural and economic context of countries in which they are implemented.

Here we share our experience and lessons learned in using different types and quality of evidence to develop guidance on the public health management of IMD for European countries within a short time period using GRADE methodology. The aim of this guidance, available on the ECDC website, was to assist countries across Europe in making decisions about appropriate measures to control and prevent IMD in contacts of cases at national and subnational levels [12].

\section{Description of the Process}

We adapted existing methods for producing evidence-based guidelines to deal with the short time frame and the scarcity of direct evidence (see Section 2.12) [10, 13-17]. The main steps are described below.

2.1. Setting Up Expert Groups. We set up a consortium of national experts: four in the area of epidemiology and public health surveillance and one in the area of microbiology of meningococcal disease. The consortium members represented five EU countries, previously involved in the assessment of national practices for IMD management across the European Union [6]. The consortium identified research questions, developed protocols, identified, assessed, and graded evidence, and formulated and graded recommendations. This work was contracted for completion within 6 months, had a budget of 20,000 Euros, and was conducted through two face-to-face meetings, three teleconferences, and close to 500 e-mail exchanges. Each member of the expert group completed a declaration of potential conflict of interests.

The consortium identified other national epidemiologists and microbiologists working with meningococcal disease from all EU countries through two established European networks (the European Meningococcal Disease Society and the ECDC European Invasive Bacterial Diseases Surveillance Network). These EU experts were asked to provide any related grey literature and technical advice during the process. Additionally, the consortium consulted two patient group networks, both based in the United Kingdom, on patientrelated values and preferences.

2.2. Defining the Area of Guidance and Formulating the Research Questions. The consortium defined research questions for guidance focused on the prevention of subsequent cases following sporadic cases of IMD and based on the needs identified through two previous surveys among public health representatives of EU countries $[6,18]$. Five research questions for evidence assessment are discussed in this paper. A summary of the evidence and recommendations for all research questions can be found in the ECDC guidance [12].

\section{Research Questions for Evidence Assessment}

(A) What is the effectiveness of chemoprophylaxis to a case of IMD before discharge from hospital in preventing further cases of IMD?

(B) What is the effectiveness of chemoprophylaxis to household contacts of an IMD case in preventing further cases?

(C) What is the effectiveness of chemoprophylaxis to contacts of an IMD case in pre-school and school settings in preventing further cases?

(D) What is the effectiveness of chemoprophylaxis to those sharing the same transport vehicle as an IMD case in preventing further cases?

(E) Which antibiotic regimes are most effective in eradicating carriage among adults, children and pregnant women?

2.3. Defining the Methodology. We opted for GRADE (Grading of Recommendations Assessment, Development, and Evaluation) methodology to assess evidence and produce guidance; we used the GRADE guidance available at the time of this study (2008-09) [14,17,19]. GRADE not only considers the balance between the benefits and harm and the quality of evidence but also includes additional factors on which to base recommendations, such as burden of the intervention on the patient, patients' values and preferences, and resource implications, which were not addressed by a number of other grading systems. GRADE also provides clear criteria to qualify the strength of a recommendation. Although GRADE has been considered by some as being too resource intensive 
and difficult to apply in public health guidance, especially under time constraint and when evidence is limited [20], it is recognized by others to provide a systematic approach, promote dialogue, and ensure documentation of the process that leads to a given recommendation [21, 22]. This makes decision-making more transparent. We referred to previous experience reported in two World Health Organization (WHO) publications on rapid advice guidelines that made use of GRADE methodology and to Cochrane guideline for public health interventions [14, 15, 23].

The consortium developed protocols, templates, and checklists for screening abstracts/papers retrieved in the literature searches to ensure a homogenous process across the research questions and across reviewers. The process was also reviewed against the criteria for guideline development as defined by the AGREE collaboration [16].

2.4. Search Strategy and Selection Criteria for Systematic Reviews. When defining the most suitable terms for the population, intervention, comparison, and outcome (PICO) to define our research questions for the search strategy (see examples in Table 1) [15], we took into account prior knowledge and a preassessment of the literature. Our preassessment suggested that measurements of direct outcomes would not be available for several research questions due to the low incidence of IMD. For these questions, we defined and included proxy outcomes in our search strategy (see examples below Section 2.5).

We defined inclusion and exclusion criteria for selecting studies, applied to each research question. All European languages were included to avoid publication biases. As most of our studied interventions either were standard clinical practice or involved rare outcomes, clinical trials had not been conducted for ethical or logistical reasons. We thus did not limit inclusion to experimental studies but also included observational studies that involved comparison groups and case series with at least 10 cases.

We searched MEDLINE, Embase, Global Health, the Cochrane database of systematic reviews, and the Cochrane central register of controlled trials. The search terms for each question were agreed by at least two members of the consortium. Due to the short time frame, we applied some of the strategies previously used in rapid reviews by limiting the search to the period from January 1990 to the date of the literature search (December 2008) and giving priority to systematic reviews [24]. If a relevant systematic review was identified, we only considered abstracts published from the date of search for the last review up to the end of 2008. If no relevant review was identified, we screened all abstracts published from 1990 to 2008 . We reviewed full papers of abstracts identified as relevant. One reviewer only was involved in reviewing abstracts and full papers of each research question due to time constraints. In case of doubts, ad hoc opinion of a second reviewer was requested.

We examined reference lists of the selected papers for other relevant publications and searched Google Scholar for citations of identified key papers. For instance, for the research question on effectiveness of antibiotic regimens
(Question E), we found a nonindexed but peer reviewed trial on antibiotic prophylaxis by using Google Scholar, though this study had not been retrieved by a previous Cochrane systematic review [25].

2.5. Use of Indirect Evidence. As studies measuring direct evidence on outcomes could not be found in four of the five research questions discussed in this paper, we defined and searched for indirect evidence on outcomes. For example, the relevant direct outcome for the question on the effectiveness of chemoprophylaxis before hospital discharge in preventing further cases among contacts (Question A) would be the incidence of subsequent cases in household contacts of the IMD patients who received antibiotics prior to discharge from hospital. A prior systematic review did not identify relevant studies measuring this outcome but showed that eradicating nasopharyngeal carriage in household contacts reduced the risk of further cases [7]. We thus searched for data on the proxy outcome, that is, the prevalence of meningococcal carriage in discharged patients (Table 1).

We also did not find direct evidence on the research question regarding whether chemoprophylaxis of contacts in school settings would prevent further cases (Question C). However, we obtained indirect evidence by comparing the risk of subsequent cases in school contacts (not receiving chemoprophylaxis) with the background incidence rates of IMD in the relevant population [26].

Even when the literature search provided direct evidence on the benefits of an intervention (e.g., effectiveness in preventing secondary cases), the evidence was often insufficient on its harm. In particular, direct evidence on the adverse events of antibiotics administered as chemoprophylaxis (Question E) was insufficient, but we found and reviewed indirect evidence on adverse events of these antibiotics when administered for indications other than chemoprophylaxis (e.g., ciprofloxacin used in cystic fibrosis).

2.6. Analysis of Extracted Data. We extracted and summarized the evidence on benefits and harm and prepared evidence profiles. When possible, we pooled estimates retrieved from selected studies. For instance, for Question B on chemoprophylaxis for household contacts, we extracted results from a recent study published after a systematic review and analysed these together with the three former studies from the review [7, 27-29]. As the results of the four studies were statistically homogeneous, we calculated a common pooled estimate (Figure 1) [12]. In case of heterogeneity between studies, we performed stratified analysis when possible. In particular, analyses on the effectiveness of chemoprophylaxis to contacts of an IMD case in preschool and school settings (Question C) were stratified by each educational setting [26].

If the retrieved systematic reviews did not provide the level of detail needed to calculate pooled estimates of effectiveness or to fully answer the research questions, we extracted the necessary data from primary studies when appropriate. For instance, in the research question on antibiotic regimes for different subgroups (Question E), we identified Cochrane systematic review on antibiotics for preventing 


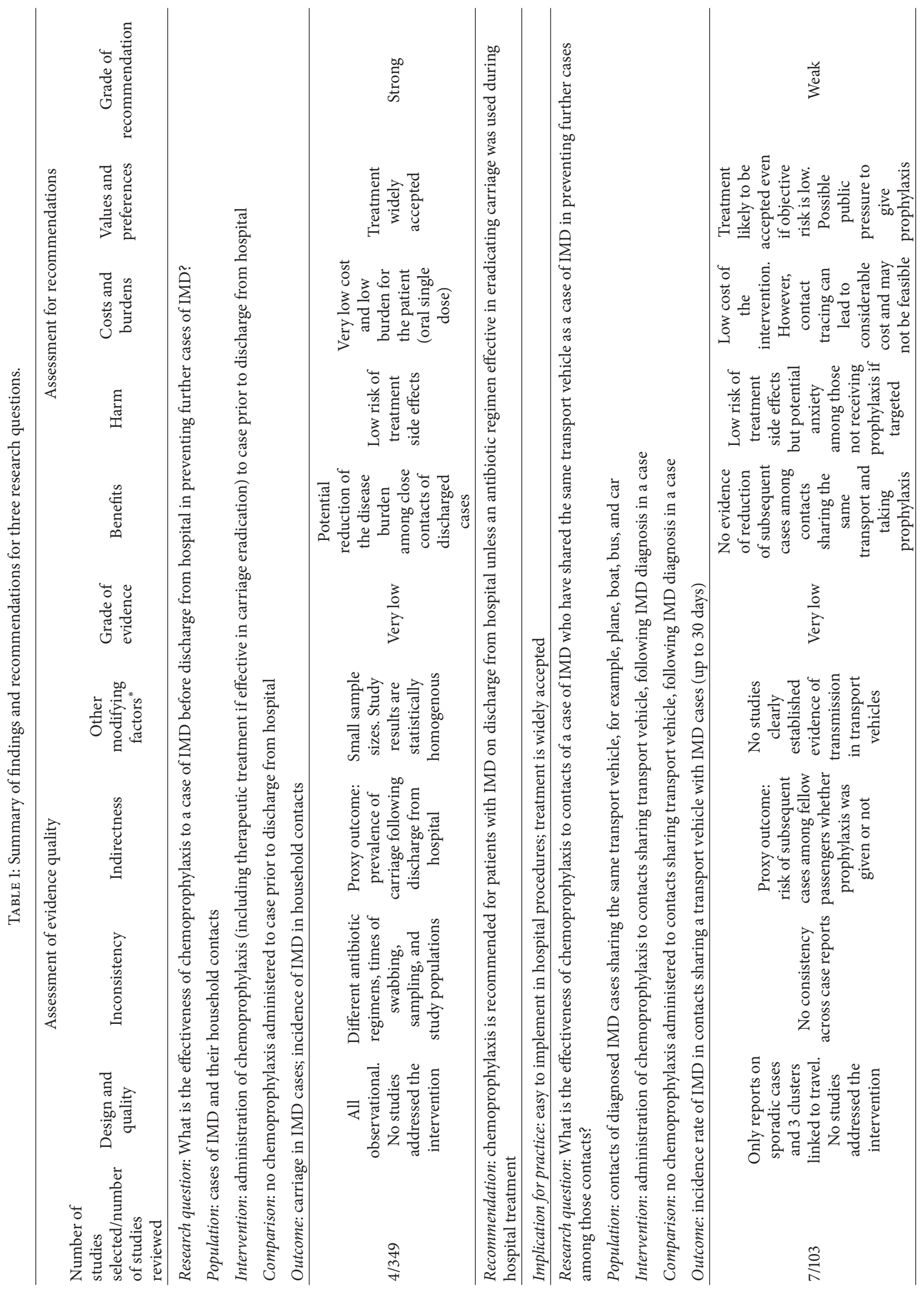




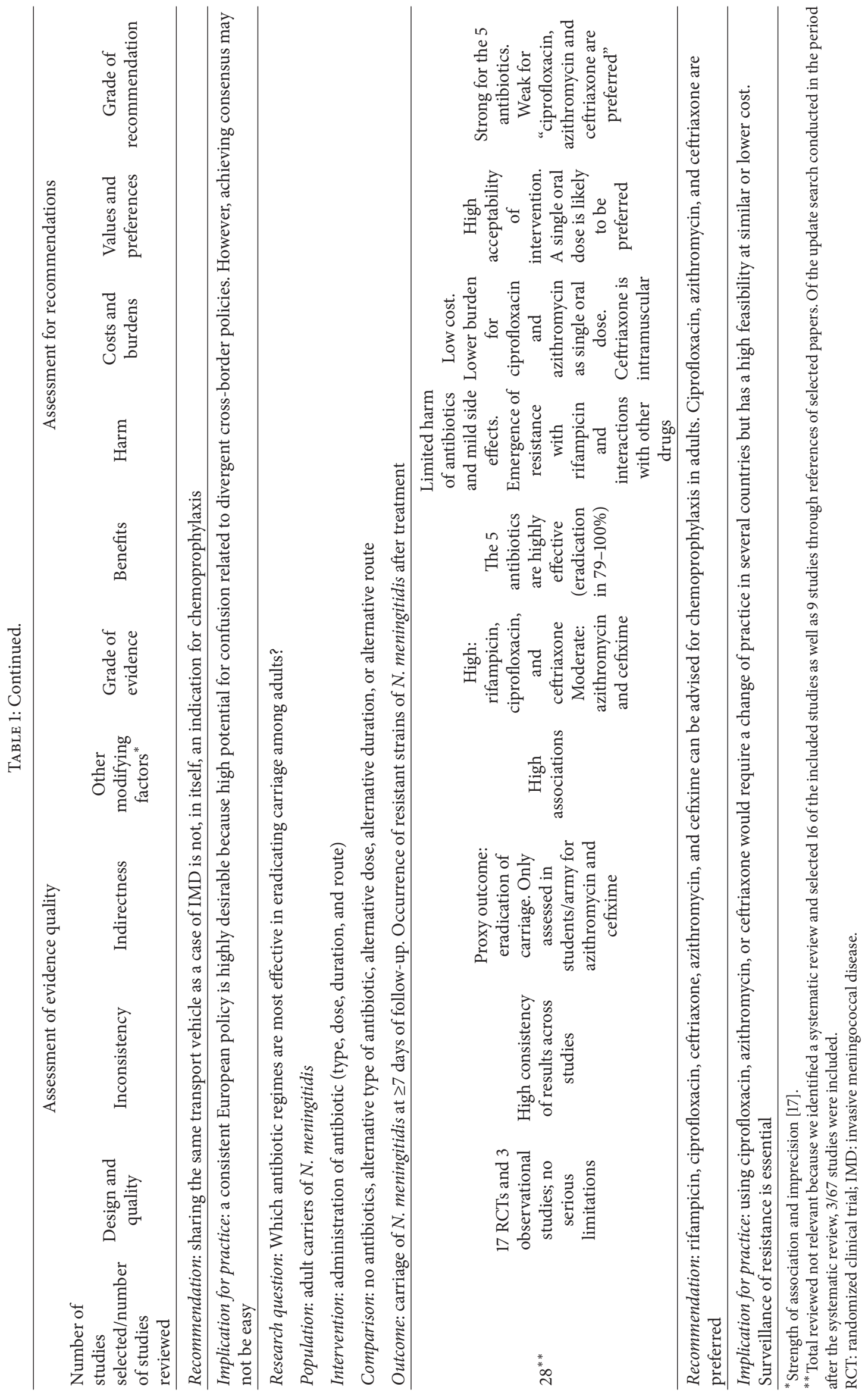




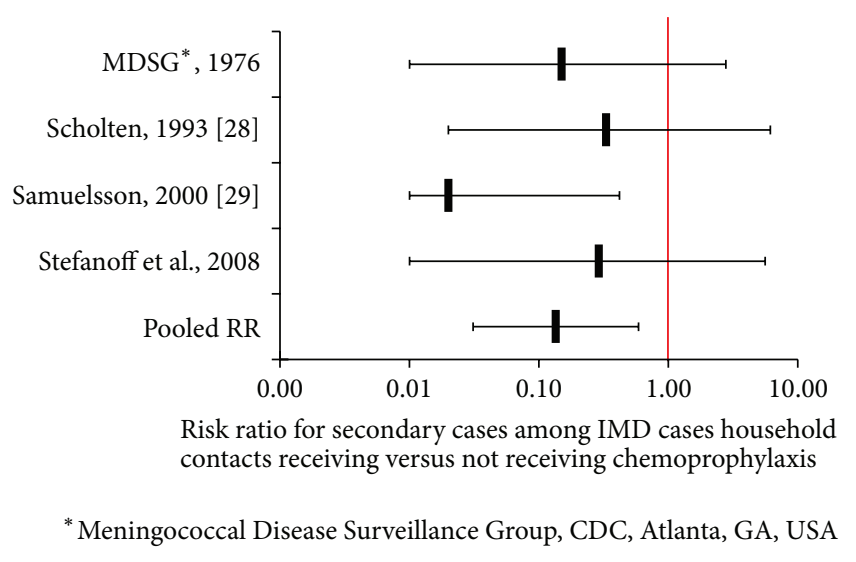

FIGURE 1: Estimate of effect of chemoprophylaxis to household contacts following a sporadic IMD case.

meningococcal infections, but this review did not present detailed analyses by antibiotic dosage and duration of therapy and did not stratify estimates by subgroups such as children and pregnant and lactating women [25]. We thus retrieved the nine primary studies that involved different dosages, treatment durations, and subgroups, extracted the required data, and appraised the studies based on the full papers.

2.7. Reviewing and Grading Evidence. Evidence was classified for all questions as either direct or indirect. We graded bodies of evidence according to GRADE guidelines and classified them as high, moderate, low, or very low, based on study design and quality, inconsistency, indirectness, imprecision, and strength of the association [17, 19]. In particular, we made a judgment of whether the evidence was sufficiently indirect to warrant downgrading. For example, we downgraded the quality of evidence for the four studies retrieved for the research question on chemoprophylaxis for cases before hospital discharge (Question A), as they measured a proxy outcome, had very small study populations ranging from 14 to 51, and used different therapeutic antibiotic regimes. As observational studies start with "low quality" evidence rating according to GRADE [13], this led to classification of the evidence quality to "very low."

We found high or moderate quality evidence for only two of the five research questions, that is, regarding chemoprophylaxis to household contacts (Question B) and antibiotic regimes (Question E). Only low or very low quality of evidence was found for the remaining three research questions (Questions A, C, and D). Table 1 describes evidence review and grading for three research questions (Questions A, D, and E) for which the quality of evidence was very low (A and D) or moderate to high (E).

2.8. Burden on Patient, Values, Preferences, and Resource Implications. Because these factors varied across settings [21, 22], we first outlined, for each question, what should be considered from the patients perspective as burden of the intervention and their values and preferences and resource implications in an EU setting. For instance, for the burden of prophylactic antibiotics (Question E), we considered for each antibiotic the potential side effects, inconvenience (e.g., number of days of treatment) for contacts, ease of administration, and the number of contacts needed to be treated (where possible to calculate) to prevent one IMD case among contacts according to each setting. We also considered the implications of contact tracing, as this can lead to considerable costs when, for instance, tracing close contacts on the same aeroplane as an IMD case is required (Question D) and may even not be feasible, for example, in case of free seating.

We found little information in the literature on burden of intervention perceived by patients and on their values and preferences. We searched for alternative data sources: for instance, information on perceived burden and values was requested from EU experts and national IMD representatives as well as from two meningitis patient associations. This confirmed that IMD is perceived as a severe disease that generates a high level of anxiety, and thus prevention measures are widely accepted, even if associated with some level of discomfort.

\subsection{Developing and Grading the Strength of Recommenda-} tions. The consortium met face-to-face to develop recommendations according to GRADE, based on the quality of evidence and the balance between the benefits and harm, taking into account burden, values, preferences, and costs (see examples in Table 1).

Recommendations were classified as strong or weak as recommended by GRADE [10,14, 17]. The GRADE guidance available at the time of developing this guidance did not provide an objective method for assessing the balance between benefit, harm, burden, values, and costs [13, 17]. We decided that the entire consortium should participate and agree on this appraisal process and we included advice of two patients' groups regarding values and preferences related to the recommendations. Based on these criteria, four strong and five weak recommendations for intervention were made for the five research questions. Strong recommendations were made not only in relation to two research questions with moderate to high quality of evidence (Questions B and E) but also in relation to one research question with very low quality of evidence (Question A). The weak recommendations for or against intervention referred not only to two research questions with low or very low quality of evidence (Questions $\mathrm{C}$ and $\mathrm{D}$ ) but also to two aspects of one research question with moderate quality of evidence (Question E).

The strong recommendation for which the quality of evidence on the benefit was very low was related to the research question on chemoprophylaxis of IMD cases before hospital discharge (Question A). Despite the very low quality evidence on the benefits, the consortium considered that harm, cost, burden, and values were strongly in favour of the intervention: the low cost of the intervention, the low number of patients not treated with an eradicating antibiotic regimen prior to discharge, and the potential benefit in reducing risk from a life-threatening disease were balanced against 
limited harm from antibiotics (Table 1). In general, higher quality evidence is more likely to be associated with strong recommendations than lower quality evidence [11]. However, the GRADE methodology indicates that a particular quality of evidence does not imply a particular strength of recommendation. A number of public health guidances and GRADE clinical guidelines issued strong recommendations in the face of a very low quality of evidence $[11,13,14$, $23,30]$. WHO also considers that strong recommendations can be made despite low or very low quality evidence in specific circumstances, as it is the net result of all relevant factors that are important [21]. For instance, WHO rapid advice guidelines for management of sporadic human infection with avian influenza A (H5N1) virus made a strong recommendation to treat $\mathrm{H} 5 \mathrm{~N} 1$ patients with oseltamivir, although the quality of the underlying evidence was rated as very low in part because of the severity of the disease [23]. A recent review highlighted that over than half (55\%) of strong recommendations in $\mathrm{WHO}$ guidelines were based on low or very low confidence in effect estimates [30]. A GRADE guidance published after our review describes five situations in which a strong recommendation is warranted despite low or very low confidence in effect estimates [31]. The most relevant to our review was "when low quality evidence suggests benefit in a life-threatening situation," as all of our recommendations aimed at preventing a life-threatening invasive infection.

Consensus on the recommendations and grading of their strength was difficult to reach regarding chemoprophylaxis in day care settings (Question C). Here, the quality of evidence was low, and divergent recommendations were in place in the consortium members' native countries. Thus each expert was probably influenced by his/her existing national policy. This highlighted that recommendations are built not only on rigorous scientific reviews but also on expert interpretation and judgment of the evidence. An advantage of the GRADE approach is to promote useful dialogue and ensure transparency by making these value judgments explicit [17, 21].

We involved stakeholders and potential users of the guidance in the final steps. As our aim was to produce guidance that could be adapted to the needs of different EU countries, the draft document was circulated through EU experts and patient groups and reviewed by representatives of EU countries in the ECDC Advisory Forum. The feedback from ECDC and EU experts on the draft report allowed useful additions to the guidance [12]. For instance, we added recommendations on use of antibiotics by lactating women on request from representatives from a patient association.

2.10. Implications for Practice. We described how the guidance would potentially change current practice in EU countries. For instance, for the research question (E) on effectiveness of antibiotic regimens in IMD prophylaxis, we described what policy changes would be required and potential obstacles to the implementation of this guidance in a EU setting, based on whether the intervention or the specific drug is available and whether the recommended regimen differs from those currently recommended. In particular, some effective dosages did not correspond to recommendations and formulations available in EU countries and would require a change in current guidance. For instance, high quality evidence was available for the effectiveness of a single dose of $750 \mathrm{mg}$ ciprofloxacin for the eradication of meningococcal carriage. However, in many countries, ciprofloxacin is recommended as a $500 \mathrm{mg}$ single dose, although the effectiveness of this lower dosage has not been assessed in a controlled trial.

2.11. Strengths of the Process. The guidance was successfully completed within six months and was approved and endorsed by ECDC in 2010 [12]. The GRADE approach allowed transparent judgments on the quality of evidence and the formulation of recommendations. Our process met most of the criteria for guideline development as defined by the AGREE collaboration (2003 version) [16]. We complied with the following criteria: definition of the scope and purpose, stakeholder involvement, rigor of development, clarity and presentation, application, and editorial independence. On the other hand, our review process did not fulfil criteria pertaining to tools for application and audit.

An advantage of GRADE process in developing public health recommendations is the integrated appraisal of related values, preferences, burden to the patient, and resource implications in addition to quality of evidence and the balance between benefits and harm. Based on GRADE 200408 guidance, we made strong recommendations for some areas in which the quality of evidence was low or very low. The long deliberations often required to arrive at final agreement of recommendations were facilitated by frequent communication, mainly by e-mail. It should be noted that GRADE work published later provides a systematic approach by describing circumstances in which a strong recommendation is warranted despite low or very low confidence in effect estimates, but these were not available at the time of developing our guidance [30, 31].

The influence of national policies on the judgment of each consortium expert to formulate recommendations (described above) was dealt with by explicitly discussing each recommendation in the entire group. One advantage of having experts from five EU countries in the consortium was also that they had knowledge of current practices and health systems when considering implications for practice of the guidance.

The development of this guidance led to the identification of areas of uncertainty and research gaps, and we identified priorities for further research in each area. It was also a unique opportunity to progress towards common European health policy. Divergent health policies may cause confusion among the public and the media. The most objective argument for common health policies consists of a systematic and transparent search for and evaluation of available evidence. In 2013, we evaluated the impact of this guidance on the recommendations for public health management of IMD in European countries and found out that $90 \%$ of the $31 \mathrm{EU}$ countries or regions found it useful at the national level and that $50 \%$ used it to update their national guidelines within the three years following the publication of the guidance [32]. 
WHO adopted a very similar process for developing evidence-based immunization recommendations, published after we initiated this work [21]. Immunization is an area of public health prevention in which the evidence may also be indirect (e.g., immunological surrogate for clinical efficacy) as in our example. GRADE was selected by WHO because it improves transparency in decision-making, promotes dialogue, and provides opportunities to reassess the evidence as required [21].

2.12. Limitations of the Process. The consortium included mostly experts in epidemiology and microbiology. It could have benefited from including clinical experts and members of patient organization groups, but the short time frame was already challenging for finalizing the project.

The limited time (6 months) and available resources imply that our literature reviews could not meet the standards of a full systematic review. In addition, we could not cover all aspects of IMD public health management.

Indeed, the comprehensive application of the GRADE methodology including exhaustive systematic reviews may require substantial resources and more time is often required for rigorous development of guidelines [24]. Thus, in this project, we applied some strategies of rapid reviews, such as focusing on existing systematic reviews, having only one expert for evidence reviews, and limiting the search period [24]. These may have introduced biases in the selection and appraisal of studies [24]. However, we enhanced our searches through inclusion of older studies, searching manually the references of retrieved studies, not restricting literature search by language or database, and asking experts for unpublished data and potentially missed studies. Additionally, though our initial search focused on updating systematic reviews, we nonetheless retrieved relevant primary studies to extract all relevant data, if they were not provided in the systematic reviews. Some authors have suggested that when the timeframe is limited, combination of electronic searching, hand searching of relevant reference lists, and consultation with experts on potentially missed articles may provide the most comprehensive results [24]. In this regard, WHO publication on rapid advice guideline - that met similar time constraints-was a particularly useful reference in helping us to ensure transparency of the process [14]. It is likely that the specialist expertise of those performing the review as well as input from other EU experts minimized the risk that relevant studies would be missed [24].

We relied mostly on systematic reviews (including one Cochrane review) for the quality appraisal of individual studies for Questions B and E [7, 25], but these described the risk of biases and not the other GRADE criteria for assigning grades of evidence [11]. In particular we did not fully appraise bodies of evidence for each outcome for imprecision, also due to limited instructions in the GRADE guidance available in 2008 [17]. New guidance published after our review describes each criterion for appraising evidence more explicitly, including imprecision [33], allowing further downgrading for indirectness, imprecision, and reporting bias [11]. We also did not explicitly define which outcomes were critical to a decision and which ones were important for grading overall quality of evidence $[24,34]$. It is likely that the strict application of the newer GRADE guidance could have led to further downgrading of the quality of evidence for some of our outcomes, although this may not have changed our recommendations.

As explained above, one of the challenges was that we only found a low quality of evidence (according to GRADE) in most areas, as evidence from RCTs was only available on the effectiveness of antibiotic regimes in eradicating carriage.

The GRADE guidance required defining the burden of the intervention to the patient as well as patients' values, preferences, and resource implications to aid in the development of recommendations. However, the GRADE guidance available at the time of developing these recommendations did not provide a methodology to collect and appraise the evidence in these areas. We found scarce information in the literature on the burden, values, and preferences surrounding interventions and limited data on cost in a few countries, and these may differ across countries. Although we questioned EU representatives and two UK-based meningococcal patient organizations, a representative survey of patients across Europe would be required for obtaining sound and representative evidence. However, we did not have the resources to initiate a multinational public survey on these issues. Furthermore, the GRADE guidance did not standardize how the data on burden and values should affect the recommendation; this is left to deliberation on the part of the decision-making group and has been criticized as a weakness of the GRADE process [20]. The updated GRADE guidance also provides a more structured way to incorporate values and preferences in the development of recommendations [31].

\section{Conclusions}

We developed evidence-based guidance on the public health management of meningococcal disease for EU countries in a short time frame and with limited resources. A number of recommendations in this guidance were based on a low quality of sometimes indirect evidence due to the impracticability of conducting clinical trials on interventions for outcomes that are rare or that have become standard practice. However, the recommendations were generated systematically and transparently, following GRADE and AGREE standards. This approach, that explicitly integrates additional criteria with the quality of evidence, can be usefully applied to the many areas of public health policy in which quality of evidence is often low or indirect. A recent survey of European countries showed that the majority found the guidance based on this process useful, about half had used the guidance to update their national recommendations, and a higher proportion of countries since 2013 compared to that in 2007 recommended evidence-based measures for IMD public health management [32].

\section{Disclosure}

The funder had no role in the design, collection, analysis, and interpretation of data, in the preparation of the paper, and in the decision to submit it for publication. 


\section{Conflict of Interests}

The authors declare that they have no conflict of interests regarding the publication of this paper.

\section{Authors' Contribution}

James M. Stuart, Germaine Hanquet, Pawel Stefanoff, Wiebke Hellenbrand, and Sigrid Heuberger designed the study, performed the literature review, and developed the guidance. Germaine Hanquet, Pawel Stefanoff, and James M. Stuart wrote the paper with substantial contributions from Wiebke Hellenbrand, Sigrid Heuberger, and Pierluigi Lopalco. All authors approved the final version. Germaine Hanquet and Pawel Stefanoff contributed equally to this paper.

\section{Acknowledgments}

The authors wish to thank the European meningococcal experts who commented on the ECDC report and provided information. The European Centre for Disease Prevention and Control (ECDC) commissioned and partly funded the development of the guidance and covered the paperprocessing charges.

\section{References}

[1] European Centre for Disease Prevention and Control, Surveillance of Invasive Bacterial Diseases in Europe, 2011, ECDC, Stockholm, Sweden, 2013.

[2] K. Perrett, W. al-Wali, C. Read, P. Redgrave, and U. Trend, "Outbreak of meningococcal disease in Rotherham illustrates the value of coordination, communication, and collaboration in management," Communicable Disease and Public Health, vol. 3, no. 3, pp. 168-171, 2000.

[3] I. Zuschneid, A. Witschi, L. Quaback et al., "Invasive meningococcal disease with fatal outcome in a Swiss student visiting Berlin," Euro Surveillance, vol. 13, no. 45, 2008.

[4] P. De Wals, L. Hertoghe, I. Borlée-Grimée et al., "Meningococcal disease in Belgium. Secondary attack rate among household, day-care nursery and pre-elementary school contacts," Journal of Infection, vol. 3, no. 1, supplement, pp. 53-61, 1981.

[5] L. Hastings, J. Stuart, N. Andrews, and N. Begg, "A retrospective survey of clusters of meningococcal disease in England and Wales, 1993 to 1995: estimated risks of further cases in household and educational settings," Communicable Disease Report. CDR Review, vol. 7, no. 13, pp. R195-R200, 1997.

[6] M. Hoek, G. Hanquet, S. Heuberger et al., "A European survey on public health policies for managing cases of meningococcal disease and their contacts," Eurosurveillance, vol. 13, no. 10, 2008.

[7] B. Purcell, S. Samuelsson, S. J. M. Hahné et al., "Effectiveness of antibiotics in preventing meningococcal disease after a case: systematic review," British Medical Journal, vol. 328, no. 7452, pp. 1339-1342, 2004.

[8] T. Rachael, K. Schubert, W. Hellenbrand, G. Krause, and J. M. Stuart, "Risk of transmitting meningococcal infection by transient contact on aircraft and other transport," Epidemiology and Infection, vol. 137, no. 8, pp. 1057-1061, 2009.
[9] J. Latham, L. Murajda, F. Forland, and A. Jansen, "Capacities, practices and perceptions of evidence-based public health in Europe," Eurosurveillance, vol. 18, no. 10, Article ID 20421, 2013.

[10] D. Atkins, M. Eccles, S. Flottorp et al., "Systems for grading the quality of evidence and the strength of recommendations I: critical appraisal of existing approaches. The GRADE Working Group," BMC Health Services Research, vol. 4, no. 1, supplement 38, 2004.

[11] H. Balshem, M. Helfand, H. J. Schünemann et al., "GRADE guidelines: 3 . Rating the quality of evidence," Journal of Clinical Epidemiology, vol. 64, no. 4, pp. 401-406, 2011.

[12] European Centre for Disease Prevention and Control, Public Health Management of Sporadic Cases of Invasive Meningococcal Disease and Their Contacts, ECDC, Stockholm, Sweden, 2010.

[13] G. H. Guyatt, A. D. Oxman, G. E. Vist et al., "GRADE: an emerging consensus on rating quality of evidence and strength of recommendations," British Medical Journal, vol. 336, no. 7650, pp. 924-926, 2008.

[14] H. J. Schünemann, S. R. Hill, M. Kakad et al., "Transparent development of the WHO rapid advice guidelines," PLoS Medicine, vol. 4, no. 5, article el19, 2007.

[15] The Cochrane Collaboration, Systematic Reviews of Health Promotion and Public Health Interventions, The Cochrane Collaboration, Melbourne, Fla, USA, 2008.

[16] AGREE Collaboration, Appraisal of Guidelines for Research and Evaluation. Instrument Training Manual, AGREE Collaboration, 2003.

[17] D. Atkins, D. Best, P. A. Briss et al., "Grading quality of evidence and strength of recommendations," British Medical Journal, vol. 328, no. 7454, pp. 1490-1494, 2004.

[18] D. Boccia, N. Andrews, S. Samuelsson, S. Heuberger, A. Perrocheau, and J. M. Stuart, "Effectiveness of different policies in preventing meningococcal disease clusters following a single case in day-care and pre-school settings in Europe," Epidemiology and Infection, vol. 134, no. 4, pp. 872-877, 2006.

[19] G. H. Guyatt, G. Vist, Y. Falck-Ytter, R. Kunz, N. Magrini, and $\mathrm{H}$. Schunemann, "An emerging consensus on grading recommendations?” Evidence-Based Medicine, vol. 11, no. 1, pp. 2-4, 2006

[20] European Centre for Disease Prevention and Control, EvidenceBased Methodologies for Public Health-How to Assess the Best Available Evidence When Time is Limited and There is Lack of Sound Evidence, ECDC, Stockholm, Sweden, 2011.

[21] P. Duclos, D. N. Durrheim, A. L. Reingold, Z. A. Bhutta, K. Vannice, and H. Rees, "Developing evidence-based immunization recommendations and GRADE," Vaccine, vol. 31, no. 1, pp. 1219, 2012.

[22] D. Matysiak-Klose, F. Ahmed, P. Duclos et al., "Report on the 1st international workshop on procedures for the development of evidence-based vaccination recommendations, Berlin, Germany, 22-23 November 2010," Vaccine, vol. 30, no. 14, pp. 23992404, 2012.

[23] H. J. Schünemann, S. R. Hill, M. Kakad et al., "WHO Rapid Advice Guidelines for pharmacological management of sporadic human infection with avian influenza A (H5N1) virus," Lancet Infectious Diseases, vol. 7, no. 1, pp. 21-31, 2007.

[24] R. Ganann, D. Ciliska, and H. Thomas, "Expediting systematic reviews: methods and implications of rapid reviews," Implementation Science, vol. 5, no. 1, article 56, 2010.

[25] A. Fraser, A. Gafter-Gvili, M. Paul, and L. Leibovici, "Antibiotics for preventing meningococcal infections," Cochrane Database of Systematic Reviews, no. 4, Article ID CD004785, 2006. 
[26] W. Hellenbrand, G. Hanquet, S. Heuberger, S. Nielsen, P. Stefanoff, and J. M. Stuart, "What is the evidence for giving chemoprophylaxis to children or students attending the same preschool, school or college as a case of meningococcal disease?” Epidemiology \& Infection, vol. 139, no. 11, pp. 1645-1655, 2011.

[27] P. Stefanoff, M. Rosinska, G. Karczewski, and A. Zielinski, "The detection of meningococcal household clusters and their prophylaxis in the changing epidemiological situation of invasive meningococcal disease in Poland, 2003-2006," Eurosurveillance, vol. 13, no. 10, 2008.

[28] R. J. P. M. Scholten, H. A. Bijlmer, J. Dankert, and H. A. Valkenburg, "Secondary cases of meningococcal disease in the Netherlands, 1989-1990, a reappraisal of chemoprophylaxis," Nederlands Tijdschrift voor Geneeskunde, vol. 137, no. 30, pp. 1505-1508, 1993.

[29] S. Samuelsson, E. T. Hansen, M. Osler, and B. Jeune, "Prevention of secondary cases of meningococcal disease in Denmark," Epidemiology and Infection, vol. 124, no. 3, pp. 433-440, 2000.

[30] P. E. Alexander, L. Bero, V. M. Montori et al., "World Health Organization recommendations are often strong based on low confidence in effect estimates," Journal of Clinical Epidemiology, vol. 67, no. 6, pp. 629-634, 2014.

[31] J. C. Andrews, H. J. Schünemann, A. D. Oxman et al., "GRADE guidelines: 15. Going from evidence to recommendationdeterminants of a recommendation's direction and strength," Journal of Clinical Epidemiology, vol. 66, no. 7, pp. 726-735, 2013.

[32] S. H. W. Vygen, W. Hellenbrand, P. Stefanoff, G. Hanquet, S. Heuberger, and J. M. Stuart, "European public health policies for managing contacts of invasive meningococcal disease cases better harmonized in 2013 than 2007," Eurosurveillance, In press.

[33] G. H. Guyatt, A. D. Oxman, R. Kunz et al., "GRADE guidelines 6. Rating the quality of evidence-imprecision," Journal of Clinical Epidemiology, vol. 64, no. 12, pp. 1283-1293, 2011.

[34] A. R. Jadad, D. J. Cook, A. Jones et al., "Methodology and reports of systematic reviews and meta-analyses: a comparison of Cochrane reviews with articles published in paper-based journals," Journal of the American Medical Association, vol. 280, no. 3, pp. 278-280, 1998. 


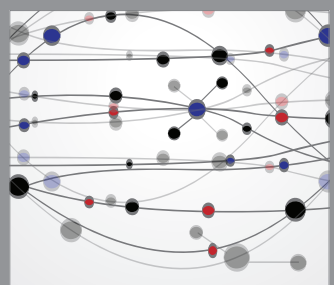

The Scientific World Journal
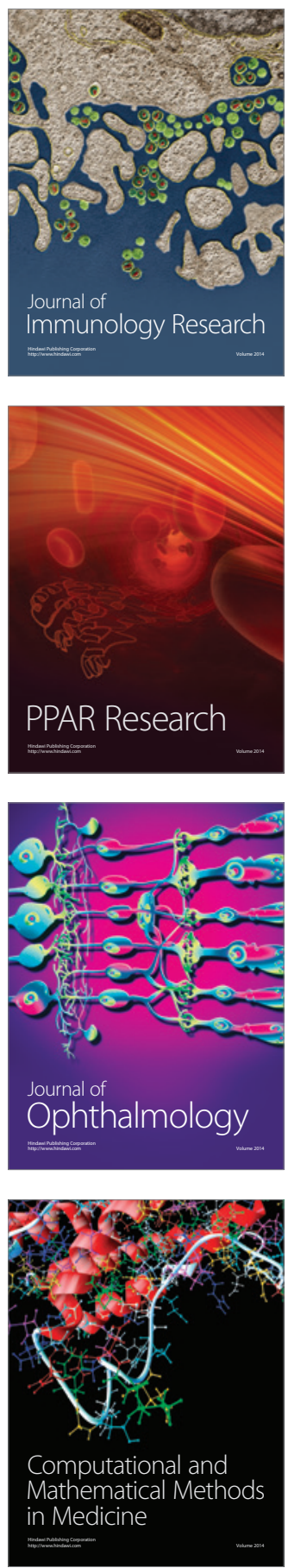

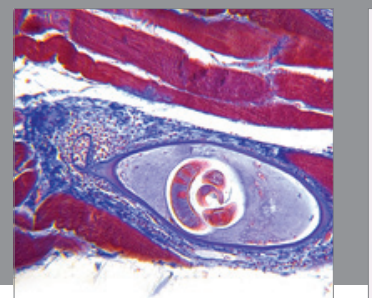

Gastroenterology

Research and Practice
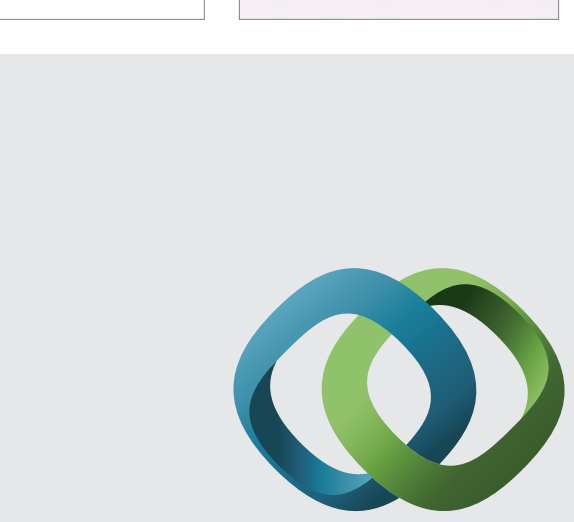

\section{Hindawi}

Submit your manuscripts at

http://www.hindawi.com
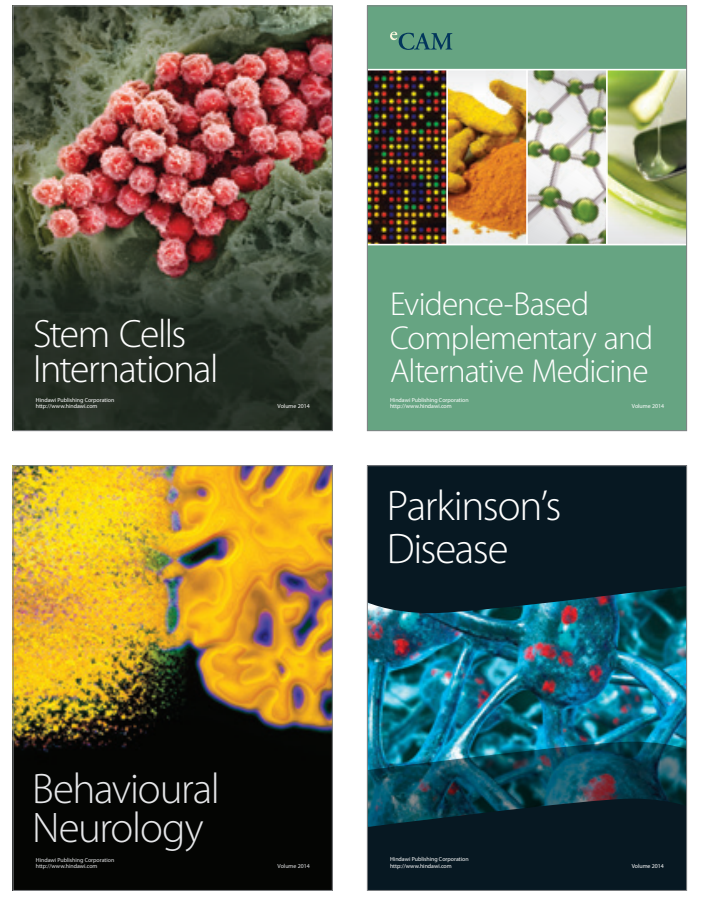
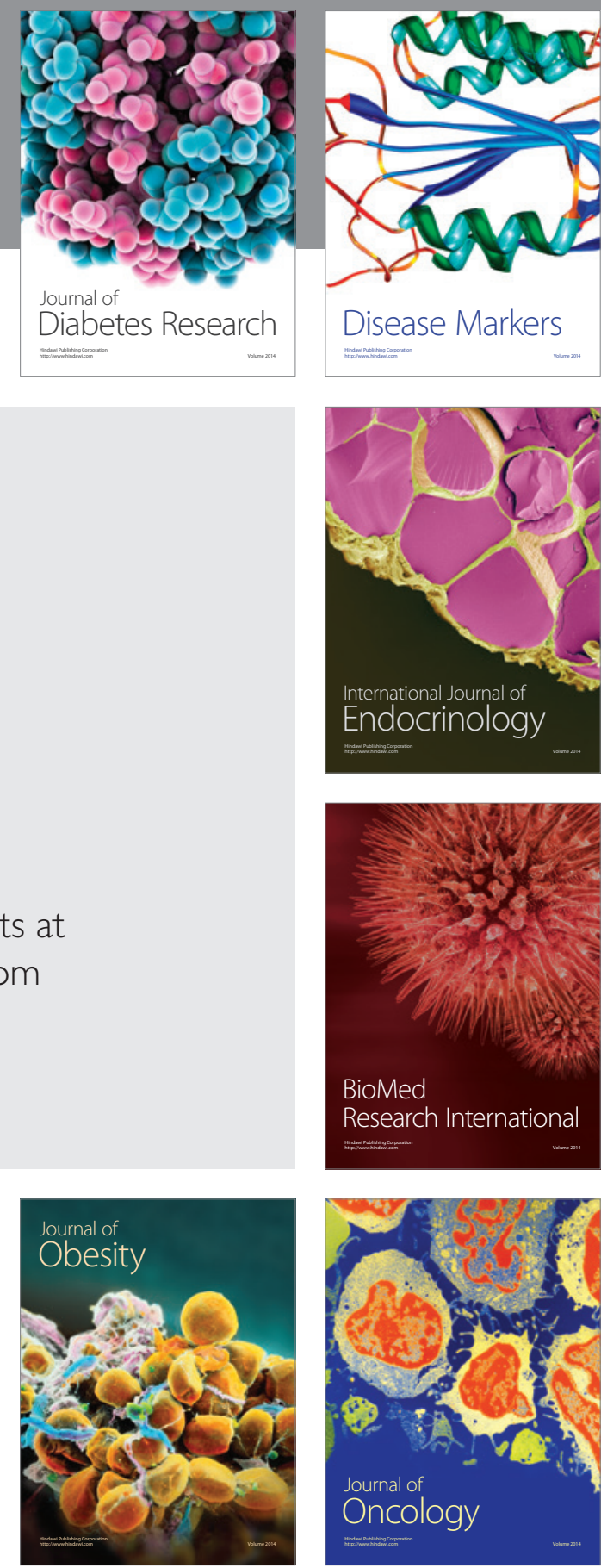

Disease Markers
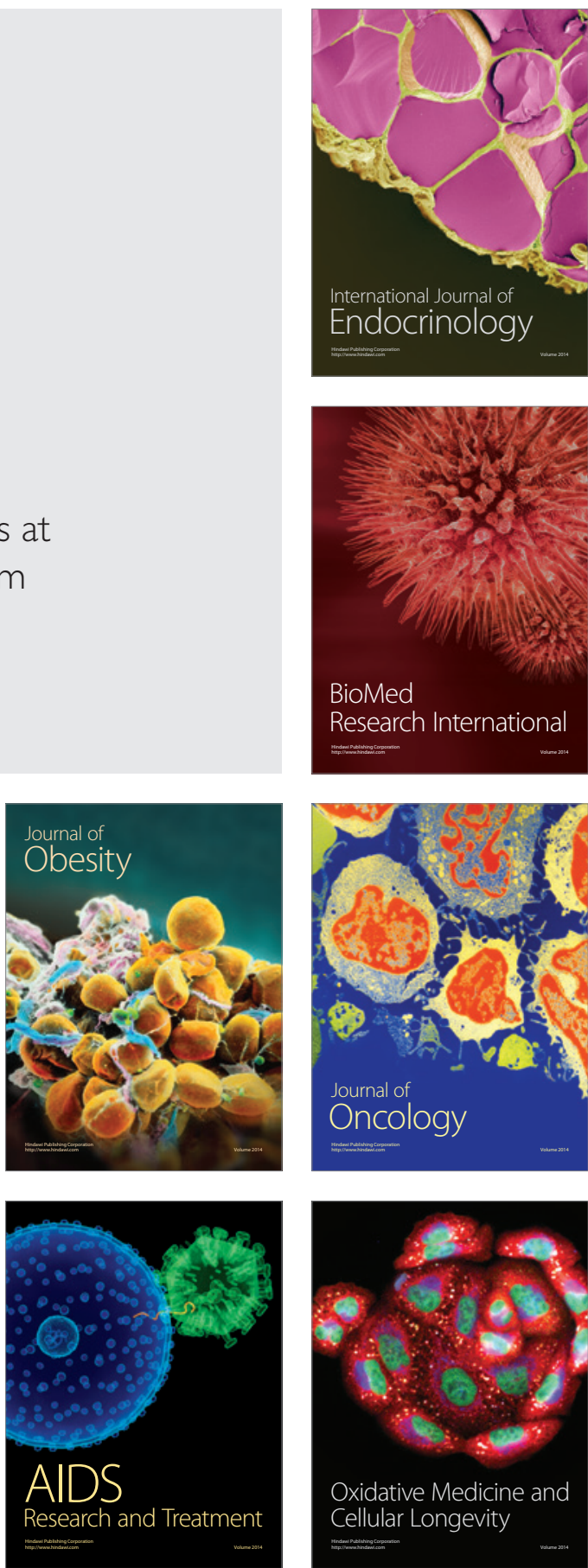